Bristol-Myers Squibb, Eisai, and Eli Lilly and Company., Speakers bureau: Bristol-Myers Squibb and Mitsubishi Tanabe Pharma., Ryuta Inaba: None declared, Tomohiro Kozuki: None declared, Masashi Taniguchi: None declared, Yuya Tabuchi Paid instructor for: Astellas Pharma, GlaxoSmithKline, Mitsubishi Tanabe Pharma, and Nippon Shinyaku., Speakers bureau: AbbVie, Janssen Pharmaceutical, Mitsubishi Tanabe Pharma, Nippon Shinyaku, and Novartis Pharma. (Outside the field of the present study.), Koji Kitagori: None declared, Syuji Akizuki: None declared, Kosaku Murakami Speakers bureau: AbbVie, Eisai, and Mitsubishi Tanabe Pharma., Ran Nakashima Grant/research support from: Takeda Pharmaceutical. (Outside the field of the present study.), Speakers bureau: Astellas Pharma, Medical \& Biological Laboratories, AstraZeneca, and Boehringer Ingelheim. (Outside the field of the present study.), Hajime Yoshifuji Grant/research support from: Astellas Pharma. (Outside the field of the present study.), Speakers bureau: Chugai Pharmaceutical. (Outside the field of the present study.), Wataru Yamamoto: None declared, Masao Tanaka Grant/research support from: AbbVie, Asahi Kasei Pharma, Astellas Pharma, Ayumi Pharmaceutical, Chugai Pharmaceutical, Eisai, Mitsubishi Tanabe Pharma, Taisho Pharmaceutical, and UCB Japan.

, Speakers bureau: AbbVie, Asahi Kasei Pharma, Astellas Pharma, Bristol-Myers Squibb, Chugai Pharmaceutical, Eisai, Eli Lilly and Company, Janssen Pharmaceutical, Mitsubishi Tanabe Pharma, Novartis Pharma, Pfizer, Taisho Pharmaceutical, Takeda Pharmaceutical, and UCB Japan., Koichiro Ohmura Grant/research support from: Astellas Pharma, AYUMI Pharmaceutical, Chugai Pharmaceutical, Daiichi Sankyo, Eisai, Japan Blood Products Organization, Mitsubishi Tanabe Pharma, Nippon Kayaku, Nippon Shinyaku, Sanofi, and Takeda Pharmaceutical., Speakers bureau: AbbVie, Actelion Pharmaceuticals Japan, Asahi Kasei Pharma, AYUMI Pharmaceutical, Bristol-Myers Squibb, Chugai Pharmaceutical, Eisai, Eli Lilly and Company, GlaxoSmithKline, Janssen Pharmaceutical, Mitsubishi Tanabe Pharma, Novartis Pharma, and Sanofi. DOI: 10.1136/annrheumdis-2020-eular.3027

\section{THU0286 THE SYSTEMIC LUPUS INTERNATIONAL COLLABORATING CLINICS (SLICC) FRAILTY INDEX (SLICC-FI) PREDICTS DAMAGE ACCRUAL IN SYSTEMIC LUPUS ERYTHEMATOSUS (SLE) PATIENTS. DATA FROM A MULTI-ETHNIC, MULTI-CENTER US LUPUS COHORT}

M. F. Ugarte-Gil ${ }^{1}, 2$, J. Dubey ${ }^{3}$, G. Mcgwin ${ }^{3}$, L. Vila ${ }^{4}$, G. S. Alarcon ${ }^{3,5} \cdot{ }^{1}$ Hospital Guillermo Almenara Irigoyen, EsSalud, Lima, Peru; ${ }^{2}$ Universidad Científica del Sur, Lima, Peru; ${ }^{3}$ University of Alabama at Birmingham, Birmingham, United States of America; ${ }^{4}$ University of Puerto Rico, Medical Sciences Campus, San Juan, Puerto Rico; ${ }^{5}$ Universidad Peruana Cayetano Heredia, Lima, Peru

Background: The Systemic Lupus International Collaborating Clinics (SLICC) Frailty Index (SLICC-FI) has been developed as a predictor of outcomes in SLE patients ${ }^{1-3}$. It combines disease activity, damage, comorbidities and health-related quality of life measures.

Objectives: To evaluate the SLICC-FI as a predictor of damage accrual in systemic lupus erythematosus (SLE) patients.

Methods: Patients from a multi-ethnic, multi-center US lupus cohort were included. Damage was ascertained with the SLICC/American College of Rheumatology (ACR) damage index (SDI) at last visit. The first visit in which the SLICC-FI could be derived was considered as the baseline visit. Univariable and multivariable Poisson regression models were performed to determine the association between the baseline SLICC-FI and last SDI, adjusted for sex, age at diagnosis, ethnicity, insurance, prednisone daily dose, antimalarial and immunosuppressive drug use at baseline. Age and gender were included a priori in the multivariable model, the other variables were included if they had a $p<0.10$ in the univariable models.

Results: Of the 503 patients included, $454(90.3 \%)$ were female with mean (SD) age 37.1 (12.5) years at diagnosis; 174 (34.6\%) were African-American, 144 (28.6\%) were Caucasians, 86 (17.1\%) Hispanics (Texas), and 99 (19.7\%) were Hispanics (Puerto Rico). The mean (SD) baseline SLICC-FI was $0.26(0.06)$. The final mean (SD) SDI score was 1.9 (2.2). Higher SLICC-FI scores at baseline predicted greater damage accrual in the univariable analysis [Estimate $=5.058$, ( $S E=0.498) ; p<0.0001]$. The SLICC-FI remained associated with damage accrual in the multivariable model, after adjustment for possible confounders [Estimate= 3.561 ( $\mathrm{SE}=0.538$ ); $\mathrm{p}<0.0001]$.

Conclusion: The SLICC-FI predicts damage accrual in SLE patients from a multi-ethnic cohort, supporting the importance of this index in the evaluation of SLE patients, combining several aspects of the disease.

References:

[1] Legge A, Kirkland S, Rockwood K, et al. Construction of a Frailty Index as a Novel Health Measure in Systemic Lupus Erythematosus. J Rheumatol. 2020; 47: 72-81

[2] Legge A, Kirkland S, Rockwood K, et al. Evaluating the Properties of a Frailty Index and Its Association With Mortality Risk Among Patients With Systemic Lupus Erythematosus. Arthritis Rheumatol. 2019; 71: 1297-107
[3] Legge A, Kirkland S, Rockwood K, et al. Prediction of Damage Accrual in Systemic Lupus Erythematosus Using the Systemic Lupus International Collaborating Clinics Frailty Index (SLICC-FI). Arthitis Rheumatol. Epub ahead of print 2019 Oct 21.

Disclosure of Interests: Manuel F. Ugarte-Gil Grant/research support from: Jannsen, Pfizer, Jyoti Dubey: None declared, Gerald McGwin: None declared, Luis Vila: None declared, Graciela S Alarcon: None declared DOI: 10.1136/annrheumdis-2020-eular.5068

\section{THU0287 \\ EVALUATION OF PREDICTIVE FACTORS OF WORSE PROGNOSIS IN LUPUS NEPHRITIS: FOCUS ON NEW PATHOGENETIC PATHWAYS}

V. Varriano ${ }^{1}$, A. Paglionico ${ }^{1}$, L. Petricca ${ }^{2}$, C. DI Mario ${ }^{1}$, M. R. Gigante ${ }^{2}$, G. Tanti ${ }^{1}$, B. Tolusso ${ }^{2}$, G. Ferraccioli ${ }^{1}$, E. Gremese ${ }^{1,2} .{ }^{1}$ Division of Rheumatology, Università Cattolica del Sacro Cuore, Rome, Italy; ${ }^{2}$ Division of Rheumatology, Fondazione Policlinico Universitario "A.Gemelli"- I.R.C.C.S., Rome, Italy

Background: cytokine dysregulation plays an important role in the pathogenesis of Lupus Nephritis (LN) representing an attractive field of research aiming to find new pathways for new targeted therapies. IL-17, IL-23 axis seems to have a great influence in the development of LN.

Objectives: to evaluate the strongest prognostic factors in a cohort of patient with LN focusing on of the impact of IL-17, IL 23 axis as new pathogenetic pathway on renal outcome.

Methods: 91 patients with active $L N$ at disease onset or disease flare were enrolled. Laboratory, immunological and disease activity data were collected at the baseline and at 6(T6),12(T12),24(T24) months and at the last follow-up(FU) 84 renal biopsies were evaluated according to ISN/RPS classification, assessing the activity and chronicity indexes and the active interstitial infiltrate using the BANFF score system. Baseline serum levels of IL-17 and IL-23 were assessed by ELISA in 37 patients.

Results: among the 84 renal biopsies evaluated $77 \%$ belonged to class III and IV according to ISN/RPS; $41,8 \%$ of patients had an active interstitial infiltrate $<5 \%$, $35.2 \%$ between $5 \%$ and $25 \%$ and $15,4 \%$ above $25 \%$. Regarding immunological data $35,2 \%$ of patients revealed a seropositivity for antiphospholipid antibodies(APL+). The median serum level of IL-17 and IL-23 were $0.12 \pm 0.15 \mathrm{pg} / \mathrm{ml}$ and $27.7 \pm 9.12 \mathrm{pg} / \mathrm{ml}$ respectively. Using the ROC curves analysis we found a cut off value of $25.89 \mathrm{pg} / \mathrm{ml}$ for IL-23 for remission at T6. Among the 10 patients with a IL-23 level above this cut-off none achieved remission at T6 and the univariate analysis shows that a serum level of IL-23 above the defined cut-off was associated with an active interstitial infiltrate $>5 \%$ at renal biopsy and with the development of persistent proteinuria. The analysis of IL-17 could not let us to find a cut off value for renal damage progression since a too much high number of patients had a null value. Nevertheless patients with more elevated serum levels of IL-17 at the baseline showed more elevated level of interstitial infiltrate at renal biopsy and a worse renal outcome overall. Finally we conducted an univariate and multivariate analysis for each renal outcome considered. We found that an inflammatory interstitial infiltrate $>5 \%$ at renal biopsy and $\mathrm{APL}+$ were associated with worse renal outcome in terms of early and persistent remission, chronic damage, persistent proteinuria, and renal flare both in univariate and multivariate analysis. Higher serum level of IL-23 was associated with persistent proteinuria, renal flare and tended to be associated to chronic renal damage and persistent renal activity.

Conclusion: interstitial inflammatory infiltrate and APL+ represent in our study the strongest predictors of worse renal outcome. An higher serum level of IL-23 was found to be a negative prognostic factor pointed out the possibility to consider the IL-17-IL 23 axis as a biomarkers of a more aggressive renal disease.

Disclosure of Interests: None declared

DOI: 10.1136/annrheumdis-2020-eular.5976

\section{THU0288 \\ CANCER RISK IN PATIENTS WITH CUTANEOUS LUPUS ERYTHEMATOSUS AND SYSTEMIC LUPUS ERYTHEMATOSUS COMPAREDTOTHE GENERAL POPULATION: A DANISH NATIONWIDE COHORT STUDY}

R. Westermann ${ }^{1}$, K. Zobbe ${ }^{2}$, R. Cordtz ${ }^{1}$, L. Dreyer ${ }^{1} .{ }^{1}$ Aalborg University Hospital, Department of Rheumatology, Aalborg, Denmark; ${ }^{2}$ The Parker Institute, BispebjergFrederiksberg Hospital, University of Copenhagen, Center for Rheumatology and Spine diseases, Rigshospitalet Gentofte, Copenhagen, Denmark

Background: Research suggesting an elevated risk of cancer among patients with Systemic Lupus Erythematosus (SLE) has increased in recent years. Yet, the size of the overall cancer risk and the risk of respective cancer sites varies. Research examining the cancer risk of Cutaneous Lupus Erythematosus (CLE) patients remains limited. Therefore, in order to further guide and monitor patients with SLE and CLE, additional research estimating the risk of cancer is needed. 